\title{
Christophe Colomb ou le fruit du hasard
}

\section{Erhard Taverna}

Dr méd., membre de la rédaction

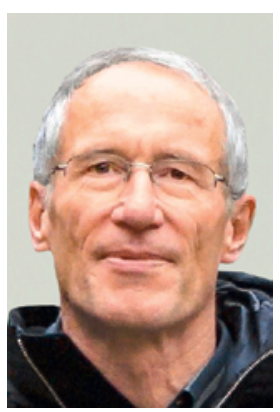

Il arrive parfois que l'on recherche quelque chose de précis et qu'on fasse une découverte tout à fait différente. Par exemple, on embarque pour l'Inde et on finit aux Caraïbes, ou encore, on cherche la pierre philosophale et on découvre la porcelaine. Une colle ratée est à l'origine du bien plus épatant Post-it. Le squelette de Scheuchzer, dont on pensait qu'il s'agissait d'une personne noyée lors du déluge, était en réalisé le fossile d'une salamandre géante. Ce que nous appelons le progrès n'est que l'interprétation de la rétrospective, l'évaluation a posteriori d'un long chemin pavé d'erreurs, de tentatives avortées, d'un carcan de réflexions traditionnelles, de superstitions et même de falsifications. Nombre d'observations médicales, par exemple concernant le cœur et la circulation sanguine, ont été mal interprétées, mais ont constitué un pas dans la bonne direction. Mais il y a également de beaux trompe-l'œil. Par exemple le réseau de canaux martiens de Schiaparelli, censés permettre de conclure à une civilisation avancée. Il existe aussi des impasses qui mènent au futur. Comme la théorie astronomique de la terre creuse de Halley censée compenser les écarts de densité. Elle a inspiré Jules Verne pour son Voyage au centre de la terre. Et même le congrès américain a financé au $19^{\mathrm{e}}$ siècle un voyage au Pôle Sud pour y chercher l'entrée supposée de ce monde merveilleux. En pleine guerre, des dirigeants nazis ont envoyé des équipes radar à Rügen pour y étudier les contours d'une chimère.

Rien ne ressort d'un système où aucune erreur n'est jamais commise et qui ronronne tranquillement. L'histoire des sciences naturelles est remplie de fausses pistes. Outre les 118 éléments recensés de la classification périodique, il y a eu de nombreuses autres découvertes avec une périodicité très courte. Même le célèbre Mendeleïev estimait qu'il existait d'autres éléments, plus légers que l'air, dont le newtonium et le coronium car il craignait que la découverte des électrons ne dévalue son système. Un livre sur les mystères ésotériques a été réédité à trois reprises, l'alabame et le virginium, «découverts» dans les années 30, ont figuré longtemps dans les manuels de chimie. En 1938, Enrico Fermi s'est vu décerner le Prix Nobel pour avoir démontré pour la première fois la fission nucléaire. Lui- même et son équipe ont considéré par erreur les produits de ce processus comme de nouveaux éléments qu'ils baptisèrent ausonium et hesperium. A part ces jolis noms, il n'en est rien resté. Sauf que, par leur travail, les auteurs ont évité à d'autres de faire fausse route. Aujourd'hui, ce sont surtout des trucages qui assurent les grands titres. Le cas du Sud-Coréen Hwang Woo-suk, dont les travaux falsifiés sur la culture de cellules souches humaines clonées ont attiré une vive attention, est exemplaire. Outre d'autres tromperies, son équipe avait fabriqué une cellule souche embryonnaire inconnue par parthénogenèse. Une icône des opposants à la vaccination, Andrew Wakefield, inquiète depuis des années de nombreux parents par ses affirmations selon lesquelles un vaccin ROR provoquerait l'autisme. Démenties depuis longtemps, ses thèses ont au moins eu un résultat politique. Elles ont aidé Donald Trump, adversaire déclaré de la vaccination, à gagner d'autres partisans pendant sa campagne électorale. Un événement qui évoque l'agronome Lyssenko soutenu par Staline, qui rejetait la doctrine génétique comme antisocialiste, et a causé par ses expérimentations de lourdes pertes de récoltes et des famines. Dans une communauté scientifique globale bien reliée, les aspects positifs des «effets Christophe Colomb» individuels ou collectifs l'emportent. Les autres doivent pouvoir vérifier ce qui est découvert ou inventé.

Souvent, les échecs accélèrent d'autres évolutions couronnées de succès. Les ballons à air chaud des frères Montgolfier ont été suivis des vols spectaculaires du Zeppelin au-dessus de l'Atlantique, jusqu'à ce que le plus grand d'entre eux connaisse une catastrophe. Tant que les humains ont cherché à imiter le vol des oiseaux, les chutes se sont succédées et ce fut un pionnier comme Otto von Lilienthal qui fit progresser l'aéronautique moderne avec ses vols planés, plutôt primitifs, bien plus que le comte Zeppelin. Il faut de tout, des fous et des visionnaires obstinés, des solitaires inadaptés défiant la routine avec leurs idées et sacrifiant ainsi leur carrière ou même leur vie. Mais le maillage d'équipes bien rodées qui non seulement renforcent leur facteur d'impact mais sont ouvertes à la nouveauté dans ce qui semble pour l'instant anormal est tout aussi indispensable. 